\title{
Genetic analysis of Wnt/PCP genes in neural tube defects
}

\author{
Zhongzhong Chen ${ }^{1,2 \dagger}$, Yunping Lei ${ }^{4 \dagger}$, Xuanye Cao ${ }^{4}$, Yufang Zheng ${ }^{1,2,3}$, Fang Wang ${ }^{6}$, Yihua Bao ${ }^{6}$, Rui Peng ${ }^{1}$, \\ Richard H. Finnell ${ }^{1,2,4,5^{*}}$, Ting Zhang ${ }^{6^{*}}$ and Hongyan Wang ${ }^{1,2,3^{*}}$ (1)
}

\begin{abstract}
Background: Mouse homozygous mutants in Wnt/planar cell polarity (PCP) pathway genes have been shown to cause neural tube defects (NTDs) through the disruption of normal morphogenetic processes critical to neural tube closure (NTC). Knockout mice that are heterozygotes of single PCP genes likely fail to produce NTD phenotypes, yet damaging variants detected in human NTDs are almost always heterozygous, suggesting that other deleterious interacting variants are likely to be present. Nonetheless, the Wnt/PCP pathway remains a genetic hotspot. Addressing these issues is essential for understanding the genetic etiology of human NTDs.

Methods: We performed targeted next-generation sequencing (NGS) on 30 NTD-predisposing Wnt/PCP pathway genes in 184 Chinese NTD cases. We subsequently replicated our findings for the CELSR1 gene in an independent cohort of 292 Caucasian NTD samples from the USA. Functional validations were confirmed using in vitro assays.

Results: CELSR1, CELSR2 and CELSR3 genes were significantly clustered with rare driver coding mutations (q-value< 0.05 ) demonstrated by OncodriveCLUST. During the validation stage, the number of rare loss of function (LoF) variants in CELSR1 was significantly enriched in NTDs compared with the LoF counts in the ExAC database $(p<0.001)$. Functional studies indicated compound heterozygote variants of CELSR2 p.Thr2026Met and DVL3 p.Asp403Asn result in down regulation of PCP signals.

Conclusions: These data indicate rare damaging variants of the CELSR genes, identified in 14\% of NTD cases, are expected to be driver genes in the Wnt/PCP pathway. Compound damaging variants of CELSR genes and other Wnt/PCP genes, which were observed in 3.3\% of the studied NTD cohort, are also expected to amplify these effects at the pathway level.
\end{abstract}

Keywords: Neural tube defects, PCP (planar cell polarity), Wnt, Variant, CELSR

\section{Background}

Neural tube defects (NTDs) resulting from the failure of neural tube closure (NTC) are among the most common and severe forms of developmental defects in humans. The complete closure of the neural tube requires the coordination of neural-plate apical constriction and convergent extension, both of which are directly regulated by the non-canonical Wnt/planar cell polarity (PCP)

\footnotetext{
* Correspondence: finnell@bcm.edu; zhangtingcv@126.com; wanghy@fudan.edu.cn

${ }^{\dagger}$ Equal contributors

'Obstetrics and Gynecology Hospital, State Key Laboratory of Genetic Engineering at School of Life Sciences, Institute of Reproduction and Development, Fudan University, Shanghai 200011, China

${ }^{6}$ Beijing Municipal Key Laboratory of Child Development and Nutriomics, Capital Institute of Pediatrics, Beijing 100020, China

Full list of author information is available at the end of the article
}

pathway [1]. Homozygous mouse mutants in the PCP pathway are known to cause NTDs through the disruption of NTC. Loss of function (LoF) alleles of the core components of the PCP pathway, including Celsr1 [2] and Vangl2 [3] produces craniorachischisis in mice. In addition, double but not single knockouts at $F z d 3$ and Fzd6 [4], as well as Dvls [5, 6] result in the NTD known as craniorachischisis in mice. In recent years, functional variants in PCP pathway genes have been identified in human NTDs, including: CELSR1 [7, 8], DVL2 [9], VANGL1 [10], VANGL2 [11], SCRIB [12], LPR6 [13] and FZD6 [14]. PCP genes appear to play an important role in the etiology of these human congenital malformations [15]. Besides these PCP components, alterations in Wnt signaling has previously been associated with NTDs [16] 
and Wnt signaling proteins also direct PCP signals in the vertebrate ectoderm [17]. The polarity of Prickle3/ Vangl2 complex was disrupted by several Wnt antagonists in the ectoderm of early Xenopus embryos; while Wnt5a, Wnt11, and Wnt11b disrupted Prickle3/Vangl2 complexes in mid-gastrula embryos leading to the development of NTDs [17]. Despite recent advances in genomics and bioinformatics, the genetic contribution of Wnt/PCP pathway genes to human NTDs requires further interrogation. Unlike mutations in many PCP genes that cause NTDs in mice, the variants detected in human NTDs are overwhelmingly heterozygous. Although knock out heterozygotes of single inactivated mouse genes does not typically produce NTD phenotypes, disruption of multiple genes in an individual could produce devastating consequences on embryonic development, as observed in mice [18]. Rare damaging variants were used in identifying interactions with birth defects, such as craniosynostosis [19] and congenital heart disease [20]. These data suggest that the interactions between rare compound damaging variants may contribute to the etiology of NTDs in humans; however, there is a paucity of compound heterozygosity data on human NTDs.

This study analyzes a panel of Wnt/PCP genes in a population based cohort of human NTD samples. We found that all three CELSR genes (CELSR1, CELSR2 and CELSR3) play driver roles in the etiology of human NTDs. In addition, this study is the first to identify compound PCP rare damaging variants combination in human NTDs, and these genetic combinations are expected to amplify effects at the pathway level and increase the risk of NTDs.

\section{Methods}

\section{Human neural tube defects}

Our study was conducted utilizing a two-stage design, and detailed diagnostic information on the patients is provided in an Additional file 1: Table S1. Subjects in the capture sequencing stage, consisting of 184 NTD subjects, were all ethnically Han Chinese, as previously described [11, 21, 22]. The Chinese NTD samples were collected from either aborted fetuses $(23.4 \pm 6.7$ weeks) or children with spina bifida ( $6.4 \pm 4.6$ years). Subjects for the CELSR1 validation study were primarily collected from Texas (292 cases, including 192 previously reported samples [7] and 100 additional samples) and the NTDs were diagnosed by trained neurosurgeons. The US NTD samples were collected from either new born or children with spina bifida with mean age 7 . $5 \pm 5.4$ years. Protocols were reviewed and approved by the Ethics Committee of the School of Life Sciences, Fudan University (EC\#216). American NTD study protocols were reviewed and approved by the Institutional Review Board (IRB) of University of Texas at Austin
(UT Austin IRB approval number: 2014120041). All of the samples were obtained with the appropriate parental informed consent.

\section{DNA sequencing, genotyping and data analysis}

Capture baits were designed to cover all coding regions of $30 \mathrm{Wnt} / \mathrm{PCP}$ pathway genes based on KEGG databases [23], and the variants were detected using targeted capture and next generation DNA sequencing (NGS). Variant calling and annotation were conducted as presented in our previously published methods [8]. Based on results from NGS, rare variants were selected to characterize the patterns of Wnt/PCP pathway genes in human NTD cases using the following criteria: (i) minor allele frequency (MAF) in NTDs < 0.01 ; (ii) coding region and splice sites were included. All variants were further annotated with the Exome Aggregation Consortium (ExAC) [24]. The functional impact of missense mutations were predicted using Sorting Intolerant From Tolerant (SIFT) [25] and Polymorphism Phenotyping version 2 (Polyphen2) [26] via VEP [27].

\section{Plasmids}

XE251 pCDNA3.1(zeo)-hDsh3-Flag (DVL3-Flag) was a gift from Randall Moon (Addgene plasmid \# 16758) [28]. Plasmid pcDNA-3.0-CELSR2-GFP(CELSR2-GFP) (Clone ID: OHu24729) was purchased from Genescript. VANGL2-HA plasmid was house made by amplification of VANGL2 cDNA and cloning the VANGL2 cDNA into a pCMV-HA plasmid (CloneTech) as previously described [11]. DVL3 c.1207G > A(p.Asp403Asn) and CELSR2 c. 6077C > T(p.Thr2026Met) were introduced into DVL3Flag and CELSR2-GFP plasmids separately by GeneArt ${ }^{\circ}$ Site-Directed Mutagenesis System (Life Technologies, Carlsbad, CA). M50 Super $8 \times$ TOPFlash was a gift from Randall Moon (Addgene plasmid \# 12456) [29].

\section{Cell lines, culture conditions and transfection}

HEK-293 T (human embryonic kidney), HeLa (human cervical cancer) were grown in DMEM (Gibco) supplemented with $10 \%$ heat-inactivated fetal bovine serum (FBS, Gibco). Cultures were maintained at $37{ }^{\circ} \mathrm{C}$ in a humidified atmosphere containing $5 \% \mathrm{CO}_{2}$. Before transfection, cells were grown to $50-70 \%$ confluency. Cell transfection was carried out using Lipofectamine 2000 (ThermoFisher) according to the manufacturer's protocol.

\section{Luciferase reporter assays}

Topflash, a TCF/LEF reporter plasmid, was used for canonical Wnt pathway signaling detection [29]. AP1 reporter plasmid was used to measure activation by Jun-kinase, a downstream effector of the non-canoncial Wnt/PCP pathway signaling $[13,30]$. For luciferase reporter assays in HEK-293 T cells, cells were transfected 
with pcDNA-3.0-Dvl3-Flag (Wildtype or Mutant), pcDNA-3.0-Celsr2-GFP (Wildtype or Mutant) and TOPFlash (Addgene plasmid ID:12456) [29] or pAP1-Luc (Agilent, Santa Clara, CA) [13] and Renilla-TK plasmid with indicated concentrations. Pools of cells were lysed with passive lysis buffer (Promega), and assayed for luciferase activity using the luciferase system (Promega). All luciferase reporter assays represent the mean \pm standard error of the mean (SEM) from three independent measurements of cell pools. Three independent replicates were performed and all statistical tests were using two tailed t-test with $P<0.05$ as the significance level.

\section{Co-immunoprecipitation assay}

DVL3-Flag and VANGL2-HA were co-transfected into HEK-293 T cells and cell lysates were immunoprecipitated with Anti-Flag Dynabeads (ThermoFisher) overnight at $4{ }^{\circ} \mathrm{C}$, and then washed three times with Lysis buffers and boiled with SDS loading buffer and subjected to Western blotting. Dvl3-Flag and Vangl2-HA were detected, as indicated.

\section{Sub-cellular localization detection}

Cells were seeded onto $20 \mathrm{~mm}$ Glass Bottom Cell Culture Dish and transfected with Celsr2-GFP, Dvl3Flag. Cells were washed in PBS prior to fixation in $4 \%$ formaldehyde. For Celsr2-GFP transfected cells, GFP was directly detected by a laser scanning confocal microscope (Leica,SP5). For Dvl3-Flag transfected cells, the cells were lysed and blocked by 1\% BSA (Sigma Aldrich) for $1 \mathrm{~h}$, after which time they were incubated overnight with 1\% BSA and washed three times with cold PBS followed by an hour exposure to a FITC conjugated secondary antibody. Immunofluorescence was visualized by the EVOS cell imaging system (ThermoFisher Scientific).

\section{Statistical analysis}

OncodriveCLUST [31], which identifies genes whose alterations tend to cluster in specific protein regions with respect to synonymous alterations [32], was used to identify driver gene mutations in the Wnt/PCP pathway in NTD cases. The Fisher's exact test was employed for CELSR1 LoF variants association analysis. Statistical analyses were performed using $\mathrm{R}$ software.

\section{Results}

Genes frequently affected by rare mutations in Wnt/PCP pathway using targeted next-generation sequencing

To better understand the genetic mechanisms underlying the etiology of human NTDs, we investigated the genetic contribution of 30 genes in the Wnt/PCP pathway. Among these $30 \mathrm{Wnt} / \mathrm{PCP}$ pathway genes, mouse mutants in 15 genes resulted in NTDs with variable phenotypes and penetrance [33]. There were 264 rare variants $(\mathrm{MAF}<0.01)$ identified in the coding region and in splice sites of $30 \mathrm{Wnt} / \mathrm{PCP}$ pathway genes in the 184 human NTD samples. Among these rare variants, 255 were single nucleotide variants (SNVs) and 9 were small insertions or deletions (indels). 62 (33.7\%) human NTDs carried 2 or more rare variants, whereas 122 (66.3\%) human NTDs had only a single rare variant in the Wnt/PCP pathway. $\mathrm{T}>\mathrm{C}$ and $\mathrm{C}>\mathrm{T}$ were the two most common transitions identified in the Wnt/PCP pathway in human NTDs. The top four genes with the highest percentage of mutations in our human NTD samples are CELSR3, EP300, CELSR1 and CELSR2 (Fig. 1). This included $17 \%$ cases with CELSR3, $17 \%$ cases with EP300, 16\% cases with CELSR1 and $16 \%$ cases with CELSR2 variants. With the exception of CELSR1, none of the other CELSR genes had previously been observed as NTD mouse candidate genes producing an abnormal phenotype when inactivated.

\section{Rare variants detected in CELSR1-3 tend to be driver genes} in Wnt/PCP pathway in human NTDs using OncodriveCLUST In order to uncover the driver genes in the Wnt/PCP pathway responsible for NTDs, OncodriveCLUST [31] was used to examine those genes with significant positional clustering of mutations. It is a useful method to identify driver genes, whose biased mutations are clustered within the protein sequence for adaptive advances during evolution. Results showed that nine genes were significantly (q-value $<0.05)$ mutated in NTD cases, including: FZD4, WNT11, CELSR1, CELSR3, NFATC4, LRP6, VANGL2, CELSR2 and PRICKLE1 (Fig. 1b). Among the top four frequently mutated genes, all CELSR genes (CELSR1, CELSR2 and CELSR3) were significantly (q-value<0.05) mutated in NTDs, while EP300 was not significantly mutated in NTD cases. Mutations in these $30 \mathrm{Wnt} / \mathrm{PCP}$ genes were frequently clustered at cadherin repeats, Wnt and strabismus domains (Additional file 2: Figure S1). Our previous study identified the missense mutation CELSR1 p.Phe870Leu in the cadherin repeat domain that induced NTDs via disruption of both the PCP pathway and canonical WNT signaling [8]. This suggested that rare mutations in CELSR genes function as mutational hotspots in the etiology of NTDs. In order to validate our findings, we re-sequenced CELSR1 in 292 NTDs (including 192 previously reported samples [7] and 100 additional samples) collected in the US. Three LoF variants (c. 5050_5051insGT [7], c.5719_5720delTG [7] and c. 4417A > $\mathrm{T}$ (p.Gln1473Ter)) were identified in the CELSR1 gene. Compared with the LoF frequency from the ExAC database (13/60693), CELSR1 LoF variants $(3 / 292)$ in NTDs were significant enriched in our NTD cohort (Fisher test, $p<0.001$ ). 


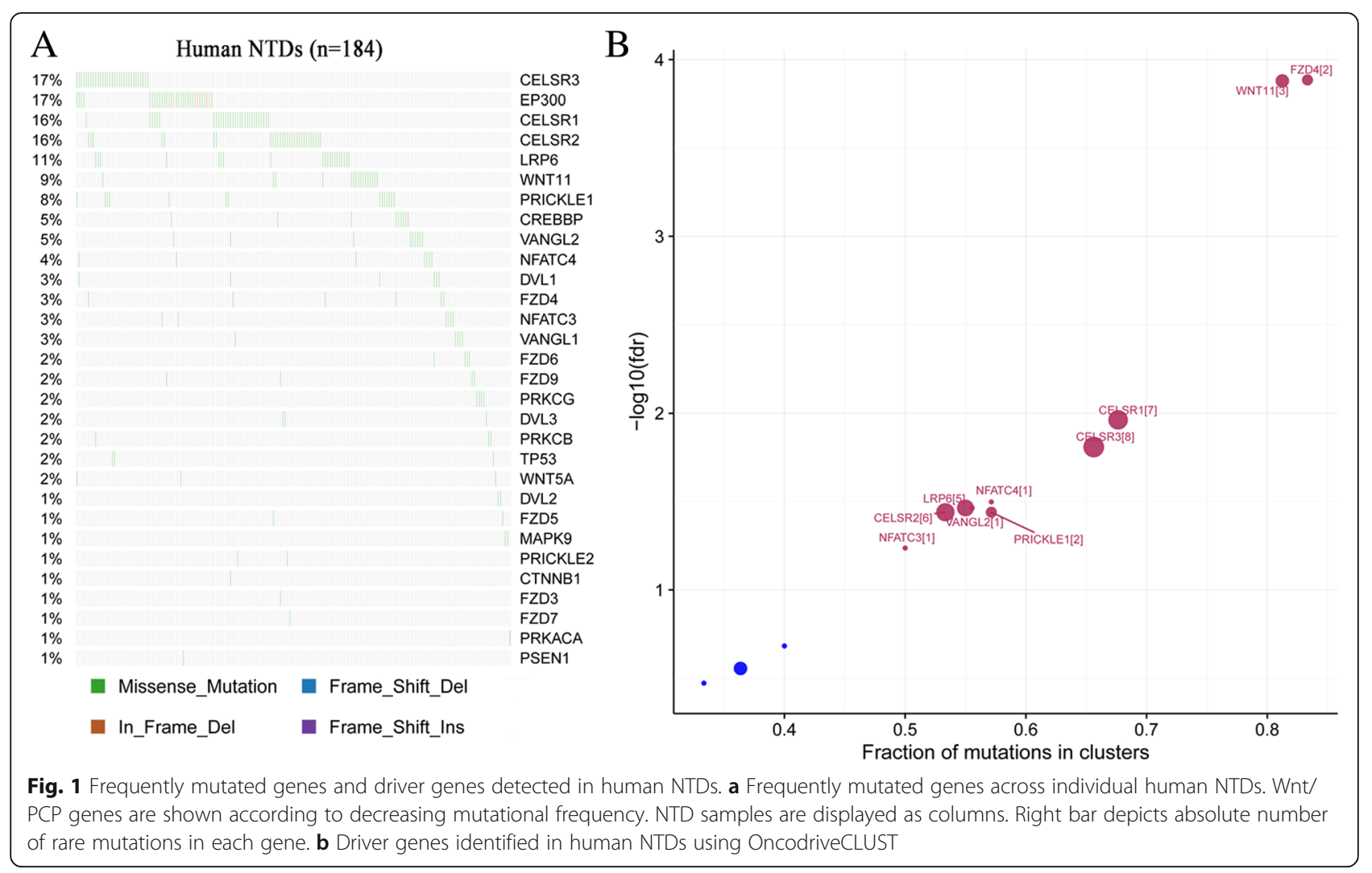

\section{Detection of double damaging variants among CELSR genes and other Wnt/PCP genes in NTD cases}

There were 27 rare mutations of CELSR genes identified in $26(14.1 \%)$ NTD cases that were predicted to be damaging variants by both PolyPhen-2 and SIFT (Fig. 2). These included eight (4.3\%) cases with damaging variants of CELSR1, nine (4.9\%) cases with damaging variants of CELSR2, and $10(5.4 \%)$ cases with damaging variants of CELSR3. In addition, six (3.3\%) cases were observed to have double Wnt/PCP heterozygous damaging mutants, and all of these cases have both damaging CELSR mutants concurrent with other Wnt/PCP mutations. These six cases were associated with diverse NTD phenotypes (Table 1). These double mutation cases included: case NTD_27 with CELSR2 p.Thr2026Met and DVL3 p.Asp403Asn, case NTD_39 with CELSR2 p. Arg2480Cys and FZD7 p.Leu383Gln, case NTD_56 with CELSR2 p.Arg2626Cys and FZD5 p.Trp242Leu, and case NTD_19 with CELSR1 p.Thr1086Met and VANGL1 p. Arg207His presented with spina bifida. Case NTD_122 with CELSR2 p.Arg1990His and CELSR3 p.Argy1194His had encephalocele. Case NTD_15 with LRP6 p. Arg386Cys and CELSR1 p.Arg714His had both anencephaly and spina bifida. Amongst the 12 damaging variants in these six cases, eight of them existed in the ExAC database with MAF $<0.001$, while four variants were novel and did not exist in the ExAC database.

\section{Functional validation of CELSR2 p.Thr2026Met and DVL3 p.Asp403Asn}

To determine the combined effect of compound heterozygosity damaging variants on PCP signaling, the functional impact of CELSR2 p.Thr2026Met and DVL3 p.Asp403Asn on canonical Wnt signaling and PCP signaling was examined in case NTD_27. These two heterozygous variants were validated by Sanger sequencing (Fig. 3a). Neither of these two variants affected the protein subcellular localization (Additional file 3: Figure S2). CLESR2 p. Thr2026Met downregulated the PCP pathway signaling in our pAP1-Luc luciferase assay (Fig. 3b). DVL3 p. Asp403Asn reduced interactions between DVL3 and VANGL2 (Fig. 3c). The DVL3 p.Asp403Asn affected both canonical and non-canonical Wnt signaling in our luciferase assay (Fig. 3d). It upregulated canonical Wnt signaling in our TopFlash assay and down regulated non-canonical Wnt/PCP signaling in our pAP1-Luc luciferase assay when $100 \mathrm{ng}$ plasmid was transfected. The two variants demonstrated a combined effect on down regulating PCP pathway signals in our pAP1-Luc luciferase assay (Fig. 3e).

\section{Discussion}

Mutations in over 300 mouse genes are associated with diverse NTD phenotypes [33], and a large number of these genes cluster in the PCP pathway [18, 34]. Recent mouse genetic studies have identified that double- 


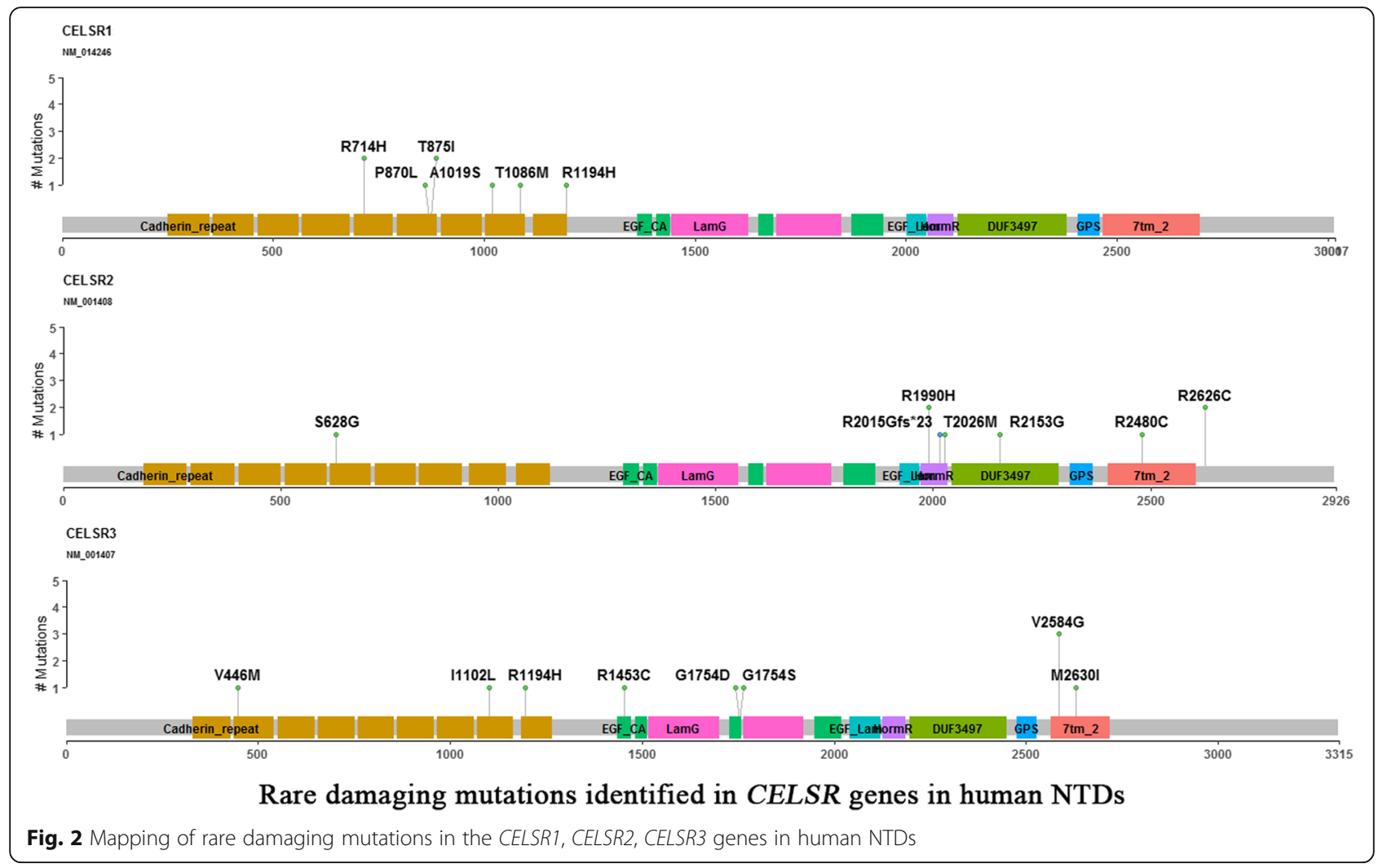

heterozygote combinations of $\operatorname{Vangl2} 2^{L P}, S c r i b^{C r c}$ and Celsr $1^{\text {Crsh }}$ are associated with a variety of NTDs, including anencephaly and spina bifida [18]. While homozygous PCP knockout mice demonstrated an NTD phenotype, single PCP heterozygote mutant mice likely fail to produce NTD phenotypes. Instead, double or multiple PCP genes in heterozygous combinations appears to be required in order to express the NTD phenotype. We propose that compound heterozygosity of genes in the PCP pathway in human populations,

Table 1 Combined rare damaging variants among Wnt/PCP genes in human NTD samples

\begin{tabular}{|c|c|c|c|c|c|c|c|c|c|c|}
\hline Sample & Gene & Variant & Chr. & Position $^{a}$ & Minor/major allele & Sex & Phenotype $^{\text {b }}$ & $\mathrm{SIFT}^{\mathrm{C}}$ & $\mathrm{PP}^{\mathrm{d}}$ & MAF in ExAC \\
\hline \multirow[t]{2}{*}{ NTD_27 } & CELSR2 & p.Thr2026Met & 1 & $109,810,233$ & $\mathrm{~T} / \mathrm{C}$ & $M$ & SB & $\mathrm{D}$ & $\mathrm{D}$ & 0.000009415 \\
\hline & DVL3 & p.Asp403Asn & 3 & $183,885,376$ & $\mathrm{~A} / \mathrm{G}$ & & & $\mathrm{D}$ & $P$ & $N A^{f}$ \\
\hline \multirow[t]{2}{*}{ NTD_39 } & CELSR2 & p.Arg2480Cys & 1 & $109,813,177$ & $\mathrm{~T} / \mathrm{C}$ & M & SB & $\mathrm{D}$ & $\mathrm{D}$ & 0.00003766 \\
\hline & FZD7 & p.Leu383Gln & 2 & $202,900,518$ & $\mathrm{~A} / \mathrm{T}$ & & & $\mathrm{D}$ & $\mathrm{D}$ & NA \\
\hline \multirow[t]{2}{*}{ NTD_122 } & CELSR2 & p.Arg1990His & 1 & $109,808,784$ & $A / G$ & M & EC & $\mathrm{D}$ & $P$ & 0.0001318 \\
\hline & CELSR3 & p.Arg1194His & 3 & $48,696,487$ & $\mathrm{~T} / \mathrm{C}$ & & & $\mathrm{D}$ & D & 0.00002825 \\
\hline \multirow[t]{2}{*}{ NTD_15 } & LRP6 & p.Arg386Cys & 12 & $12,334,194$ & $\mathrm{~A} / \mathrm{G}$ & $\mathrm{F}$ & $A E, S B$ & $\mathrm{D}$ & D & 0.000273 \\
\hline & CELSR1 & p.Arg714His & 22 & $46,930,927$ & $\mathrm{~T} / \mathrm{C}$ & & & $\mathrm{D}$ & D & NA \\
\hline \multirow[t]{2}{*}{ NTD_56 } & CELSR2 & p.Arg2626Cys & 1 & $109,814,294$ & $\mathrm{~T} / \mathrm{C}$ & NA & SB & D & $P$ & 0.0004425 \\
\hline & FZD5 & p.Trp242Leu & 2 & $208,632,739$ & $\mathrm{~A} / \mathrm{C}$ & & & D & D & NA \\
\hline \multirow[t]{2}{*}{ NTD_19 } & VANGL1 & p.Arg207His & 1 & $116,206,697$ & $\mathrm{~A} / \mathrm{G}$ & $\mathrm{F}$ & SB & D & D & 0.00001883 \\
\hline & CELSRI & p.Thr1086Met & 22 & $46,929,811$ & $\mathrm{~A} / \mathrm{G}$ & & & D & D & 0.0001318 \\
\hline
\end{tabular}

${ }^{\text {a }}$ Positions are given in bp from GRCh37

${ }^{\mathrm{b}} \mathrm{SB}$, spinabifida; $\mathrm{EC}$, encephalocele; $\mathrm{AE}$, anencephaly

'SIFT predictions: $\mathrm{D}$, deleterious

${ }^{d}$ PolyPhen2 (PP2) predictions: D, probably damaging; P, possibly damaging

${ }^{\mathrm{e}} \mathrm{MAF}$ from Exome Aggregation Consortium (ExAC) database

${ }^{f}$ Not available 


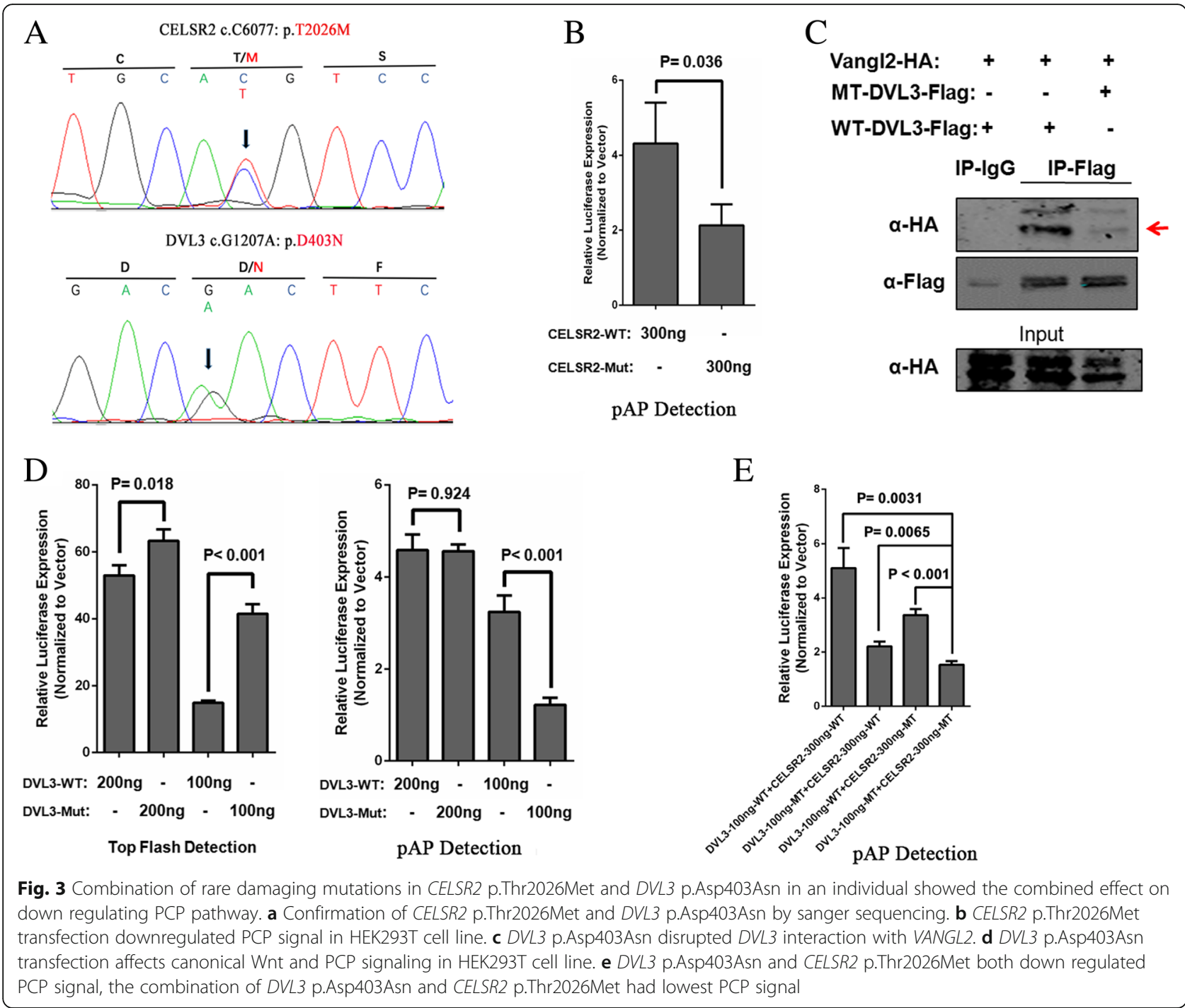

where multiple PCP gene functional variants work together in the heterozygous state, can result in NTDs. Previously reported human NTDs with missense PCP mutations were all single heterozygotes, suggesting that the 'second' or 'the other' deleterious interacting variant might be present [15]. Understanding the genetic mechanisms of Wnt/PCP pathway is essential for appreciating the genetic architecture of human NTDs. In this study, we focused on the genetic hotspots of rare coding mutants in the Wnt/PCP pathway genes, in regard to determining their genetic impact on susceptibility to NTDs. Overall, rare coding mutations were observed to be frequently mutated in CELSR 1-3 genes. Moreover, we identified 84 (46\%) cases with rare missense mutations, and $26(14.1 \%)$ cases with rare damaging missense mutations in CELSR genes, respectively. Importantly, we determined that six (3.3\%) of these cases had both damaging CELSR mutations and additional deleterious mutations of other Wnt/PCP genes, which were associated with diverse NTD phenotypes in humans.

These findings suggest that rare mutations of CELSR genes are identified as driver genes in human NTDs. CELSR, or Flamingo (Fmi) genes are a special subgroup of adhesion $G$ protein-coupled receptors (GPCRs) that have three homologs, CELSR1, CELSR2 and CELSR3. CELSR genes were first identified in Drosophila for regulating planar cell polarity in the wing [35]. Robinson and co-workers detected missense variants in CELSR1 increased risk of craniorachischisis in humans [36]. Allache and co-workers identified pathogenic CELSR1 mutations in $2.9 \%$ of Italian and Canadian NTD cohort [37]. Lei and colleagues detected deleterious CELSR1 variants in about $3 \%$ of spina bifida cases collected in the U.S. cohort [7]. In contrast to CELSR1, the genetic evidence of CELSR2 and CELSR3 contributing to NTD 
risk was previously lacking. Our previous study identified the missense mutation CELSR1 p.Phe870Leu in the cadherin repeat domain induced NTDs via disruption of both the PCP pathway and canonical WNT signaling [8]. It is implied that CELSR2 and CELSR3, clustered at cadherin repeat domain, may also contribute the human NTD risk. In the present study, we found that CELSR2 p.Thr2026Met, which is located in the HormR domain, affected PCP signaling in the HEK293T cell line (Fig. 3). Taken together, we found that in addition to CELSR1, both CELSR2 and CELSR3 also contribute to genetic etiology of human NTDs.

Previous studies reported that the damaging mutations of CELSR1 are associated with diverse NTD phenotypes in humans, such as craniorachischisis [36] and spina bifida [7]. Given the different phenotypes, these data suggests that human NTDs could be caused by compound mutant heterozygosity. Celsr 2 or Celsr 3 regulate forebrain wiring in a Vangl-independent manner and inactivation of Celsr 2 or Celsr3 produces abnormal brain morphology but does not produce NTDs in mice [38], also implicating that other unidentified molecules may participate in the genetic interactions leading to human NTDs. The present study further supported this notion by examining the damaging mutations of CELSR genes and other Wnt/PCP genes. Six (3.3\%) of NTD cases were identified to have double damaging variants that included CELSR genes and other Wnt/PCP genes, such as CELSR2 p.Thr2026Met and DVL3 p.Asp403Asn. This combination was determined to have the lowest PCP signal in vitro (Fig. 3). In addition, among these eight genes involved in the double damaging heterozygous mutations combinations (Table 1), damaging variants of the CELSR1 [7, 8], VANGL1 [10], LPR6 [13] and Dvl3 [5] are known to be associated with NTDs, indicating that compound heterozygote damaging variants, LRP6 p. Arg386Cys and CELSR1 p.Arg714His (case NTD_15), CELSR1 p.Thr1086Met and VANGL1 p.Arg207His (case NTD_19) may increase human NTD risk. As other combinations of double damaging mutations might also expand the genetic risk factors potentially associated with NTDs, further experiments are warranted to assess their contribution to the risk for human NTDs. Altogether, we added to our understanding of the role of CELSR2 and CELSR3 in human NTDs, and established that the combination of rare damaging mutations in CELSR genes and other Wnt/PCP variants in an individual could increase human NTD risk. Different combinations are associated with diverse NTD phenotypes, including spina bifida, encephaloceles, and the combined phenotype of anencephaly and spina bifida (Table 1). Our findings suggested that combinations of multiple deleterious heterozygous mutations in individuals might better explain the etiology of NTDs in humans. There are two major limitations in this study. First, we found CELSR genes as the driver genes in NTDs using OncoDriveClust [31] that is often used for identifying cancerdriving genes. Although all the compound heterozygote mutants found contain the CELSR genes, suggesting that CELSR genes are possibly the genetic hotspots in NTDs, the validity of this method needs further evaluation in human NTD cases. Second, further studies of whole genome or exome sequencing with larger sample sizes are warranted to better appreciate the genetic contributions of CELSR genes and additional PCP gene-gene interaction in the development of NTDs.

\section{Conclusions}

In conclusion, this study demonstrates that damaging mutations of CELSR genes are genetic hotspots in the development of NTDs in our human population. We also emphasized that the increased risk of double damaging variants of CELSR genes and other Wnt/PCP genes, which was observed in $3.3 \%$ of our human NTD samples. The variable outcomes of mutations in CELSR genes that interact with other Wnt/PCP genes are direct evidence linking these genes to the etiology of human NTDs, indicating that multiple genetic mutations are important for understanding the genetic etiology of human NTDs.

\section{Additional files}

Additional file 1: Table S1. Demographic characteristics in NTD cohorts. (DOCX $17 \mathrm{~kb}$ )

Additional file 2: Figure S1. Most frequent Pfam domains affected by Wnt/PCP genes in human NTD samples. (TIFF 2976 kb)

Additional file 3: Figure S2. CELSR2 p.Thr2026Met and DVL3 p.Asp403Asn did not affect the protein subcellular localization. (A) CELSR2 p.Thr2026Met did not affect CELSR2 subcellular localization in HEK293T \& MDCKII cells transfected with CELSR2-GFP and CELSR2 (p.Thr2026Met)-GFP expression plasmids. (B) DVL3 p.Asp403Asn did not affect DVL3 subcellular localization in HEK293T. (TIFF 4540 kb)

\section{Acknowledgements}

The authors would like to thank Dr. Robert M. Cabrera, Baylor College of Medicine, Houston, Texas, USA for valuable scientific input.

\section{Funding}

This work was supported by grants from the National Key Basic Research Program of China (2016YFC1000502), the National Natural Science Foundation of China $(81430005,31521003,81741048)$, and the Commission for Science and Technology of Shanghai Municipality (17JC1400902) to H. Wang and Y. Zheng. R. Finnell was supported by a Changjiang Scholar award and NIH grants HD081216 and HD067244 and March of Dimes grant \#6-FY16-169. This work is also supported by the CAMS Initiative for Innovative Medicine (2016-I2M-1-008) to T. Zhang. The funders had no role in study design, data collection and analysis, decision to publish, or preparation of the manuscript.

\section{Availability of data and materials}

The datasets analyzed during the current study are available from the corresponding author on reasonable request. 


\section{Authors' contributions}

HW, TZ and RF directed and designed the study. ZC performed the statistical and bioinformatics analyses. YL, XC, FW, YB and RP performed the experimental analysis. HW, ZC, YL, YZ and RF prepared the manuscript. All authors read and approved the final manuscript.

\section{Ethics approval and consent to participate}

Chinese NTD study protocols were reviewed and approved by the Ethics Committee of the School of Life Sciences, Fudan University (EC\#216). American NTD study protocols were reviewed and approved by the Institutional Review Board (IRB) of University of Texas at Austin (UT Austin IRB approval number: 2014120041). All of these samples were obtained with the approved of parental informed consents.

\section{Consent for publication}

Not applicable.

\section{Competing interests}

The authors declare that they have no competing interests.

\section{Publisher's Note}

Springer Nature remains neutral with regard to jurisdictional claims in published maps and institutional affiliations.

\section{Author details}

'Obstetrics and Gynecology Hospital, State Key Laboratory of Genetic Engineering at School of Life Sciences, Institute of Reproduction and Development, Fudan University, Shanghai 200011, China. ${ }^{2}$ Key Laboratory of Reproduction Regulation of NPFPC, Collaborative Innovation Center of Genetics and Development, Fudan University, Shanghai 200032, China. ${ }^{3}$ Children's Hospital and Institutes of Biomedical Sciences of Fudan University, Shanghai, China. ${ }^{4}$ Departments of Molecular and Cellular Biology and Medicine, Baylor College of Medicine, Houston, TX 77030, USA. ${ }^{5}$ Department of Pediatrics, Dell Pediatric Research Institute, University of Texas at Austin Dell Medical School, Austin, TX 78723, USA. ${ }^{6}$ Beijing Municipal Key Laboratory of Child Development and Nutriomics, Capital Institute of Pediatrics, Beijing 100020, China.

Received: 16 November 2017 Accepted: 19 March 2018 Published online: 04 April 2018

\section{References}

1. Nishimura T, Honda H, Takeichi M. Planar cell polarity links axes of spatial dynamics in neural-tube closure. Cell. 2012;149(5):1084-97.

2. Curtin JA, Quint E, Tsipouri V, Arkell RM, Cattanach B, Copp AJ, Henderson DJ, Spurr N, Stanier P, Fisher EM, et al. Mutation of Celsr1 disrupts planar polarity of inner ear hair cells and causes severe neural tube defects in the mouse. Curr Biol. 2003;13(13):1129-33.

3. Greene ND, Gerrelli D, Van Straaten HW, Copp AJ. Abnormalities of floor plate, notochord and somite differentiation in the loop-tail (Lp) mouse: a model of severe neural tube defects. Mech Dev. 1998;73(1):59-72.

4. Wang Y, Guo N, Nathans J. The role of Frizzled3 and Frizzled6 in neural tube closure and in the planar polarity of inner-ear sensory hair cells. J Neurosci. 2006;26(8):2147-56.

5. Etheridge SL, Ray S, Li S, Hamblet NS, Lijam N, Tsang M, Greer J, Kardos N, Wang J, Sussman DJ, et al. Murine dishevelled 3 functions in redundant pathways with dishevelled 1 and 2 in normal cardiac outflow tract, cochlea, and neural tube development. PLoS Genet. 2008;4(11):e1000259.

6. Wang J, Hamblet NS, Mark S, Dickinson ME, Brinkman BC, Segil N, Fraser SE, Chen P, Wallingford JB, Wynshaw-Boris A. Dishevelled genes mediate a conserved mammalian PCP pathway to regulate convergent extension during neurulation. Development. 2006;133(9):1767-78.

7. Lei Y, Zhu H, Yang W, Ross ME, Shaw GM, Finnell RH. Identification of novel CELSR1 mutations in spina bifida. PLoS One. 2014;9(3):e92207.

8. Qiao X, Liu Y, Li P, Chen Z, Li H, Yang X, Finnell RH, Yang Z, Zhang T, Qiao $B$, et al. Genetic analysis of rare coding mutations in CELSR1-3 in Chinese congenital heart and neural tube defects. Clin Sci (Lond). 2016;

9. De Marco P, Merello E, Consales A, Piatelli G, Cama A, Kibar Z, Capra V. Genetic analysis of disheveled 2 and disheveled 3 in human neural tube defects. J Mol Neurosci. 2013;49(3):582-8.
10. Kibar Z, Torban E, McDearmid JR, Reynolds A, Berghout J, Mathieu M, Kirillova I, De Marco P, Merello E, Hayes JM, et al. Mutations in VANGL1 associated with neural-tube defects. N Engl J Med. 2007;356(14):1432-7.

11. Lei YP, Zhang T, Li H, Wu BL, Jin L, Wang HY. VANGL2 mutations in human cranial neural-tube defects. N Engl J Med. 2010;362(23):2232-5.

12. Lei Y, Zhu H, Duhon C, Yang W, Ross ME, Shaw GM, Finnell RH. Mutations in planar cell polarity gene SCRIB are associated with spina bifida. PLoS One. 2013;8(7):e69262.

13. Lei Y, Fathe K, McCartney D, Zhu H, Yang W, Ross ME, Shaw GM, Finnell RH. Rare LRP6 variants identified in spina bifida patients. Hum Mutat. 2015;36(3):342-9.

14. De Marco P, Merello E, Rossi A, Piatelli G, Cama A, Kibar Z, Capra V. FZD6 is a novel gene for human neural tube defects. Hum Mutat. 2012;33(2):384-90.

15. Juriloff DM, Harris MJ. A consideration of the evidence that genetic defects in planar cell polarity contribute to the etiology of human neural tube defects. Birth Defects Res A Clin Mol Teratol. 2012;94(10):824-40.

16. Carter M, Chen X, Slowinska B, Minnerath S, Glickstein S, Shi L, Campagne F, Weinstein H, Ross ME. Crooked tail (cd) model of human folate-responsive neural tube defects is mutated in Wnt coreceptor lipoprotein receptorrelated protein 6. Proc Natl Acad Sci U S A. 2005;102(36):12843-8.

17. Chu CW, Sokol SY. Wnt proteins can direct planar cell polarity in vertebrate ectoderm. elife. 2016:5

18. Murdoch JN, Damrau C, Paudyal A, Bogani D, Wells S, Greene ND, Stanier P, Copp AJ. Genetic interactions between planar cell polarity genes cause diverse neural tube defects in mice. Dis Model Mech. 2014;7(10):1153-63.

19. Timberlake AT, Choi J, Zaidi S, Lu Q, Nelson-Williams C, Brooks ED, Bilguvar K, Tikhonova I, Mane S, Yang JF, et al. Two locus inheritance of non-syndromic midline craniosynostosis via rare SMAD6 and common BMP2 alleles. elife. 2016;5

20. Homsy J, Zaidi S, Shen Y, Ware JS, Samocha KE, Karczewski KJ, DePalma SR, McKean D, Wakimoto H, Gorham J, et al. De novo mutations in congenital heart disease with neurodevelopmental and other congenital anomalies. Science. 2015;350(6265):1262-6.

21. Shi $Y$, Ding $Y$, Lei $Y P$, Yang $X Y$, Xie GM, Wen J, Cai CQ, Li H, Chen Y, Zhang $T$, et al. Identification of novel rare mutations of DACT1 in human neural tube defects. Hum Mutat. 2012;33(10):1450-5.

22. Yang $X Y$, Zhou $X Y$, Wang QQ, Li H, Chen Y, Lei YP, Ma XH, Kong P, Shi Y, Jin L, et al. Mutations in the COPII vesicle component gene SEC24B are associated with human neural tube defects. Hum Mutat. 2013;34(8):1094-101.

23. Kanehisa M, Furumichi M, Tanabe M, Sato Y, Morishima K. KEGG: new perspectives on genomes, pathways, diseases and drugs. Nucleic Acids Res. 2017:45(D1):D353-61.

24. Lek M, Karczewski KJ, Minikel EV, Samocha KE, Banks E, Fennell T, O'DonnellLuria AH, Ware JS, Hill AJ, Cummings BB, et al. Analysis of protein-coding genetic variation in 60,706 humans. Nature. 2016;536(7616):285-91.

25. Kumar P, Henikoff S, Ng PC. Predicting the effects of coding nonsynonymous variants on protein function using the SIFT algorithm. Nat Protoc. 2009;4(7):1073-81.

26. Adzhubei IA, Schmidt S, Peshkin L, Ramensky VE, Gerasimova A, Bork P, Kondrashov AS, Sunyaev SR. A method and server for predicting damaging missense mutations. Nat Methods. 2010;7(4):248-9.

27. McLaren W, Pritchard B, Rios D, Chen Y, Flicek P, Cunningham F. Deriving the consequences of genomic variants with the Ensembl API and SNP effect predictor. Bioinformatics. 2010;26(16):2069-70.

28. Angers S, Thorpe CJ, Biechele TL, Goldenberg SJ, Zheng N, MacCoss MJ Moon RT. The KLHL12-Cullin-3 ubiquitin ligase negatively regulates the Wnt-beta-catenin pathway by targeting Dishevelled for degradation. Nat Cell Biol. 2006;8(4):348-57.

29. Veeman MT, Slusarski DC, Kaykas A, Louie SH, Moon RT. Zebrafish prickle, a modulator of noncanonical Wnt/Fz signaling, regulates gastrulation movements. Curr Biol. 2003;13(8):680-5.

30. Allache R, Lachance S, Guyot MC, De Marco P, Merello E, Justice MJ, Capra V, Kibar Z. Novel mutations in Lrp6 orthologs in mouse and human neural tube defects affect a highly dosage-sensitive Wnt non-canonical planar cell polarity pathway. Hum Mol Genet. 2014;23(7):1687-99.

31. Tamborero D, Gonzalez-Perez A, Lopez-Bigas N. OncodriveCLUST: exploiting the positional clustering of somatic mutations to identify cancer genes. Bioinformatics. 2013;29(18):2238-44

32. Gonzalez-Perez A, Perez-Llamas C, Deu-Pons J, Tamborero D, Schroeder MP, Jene-Sanz A, Santos A, Lopez-Bigas N. IntOGen-mutations identifies cancer drivers across tumor types. Nat Methods. 2013;10(11):1081-2.

33. Wilde JJ, Petersen JR, Niswander L. Genetic, epigenetic, and environmental contributions to neural tube closure. Annu Rev Genet. 2014;48:583-611. 
34. Wu G, Huang X, Hua Y, Mu D. Roles of planar cell polarity pathways in the development of neural [correction of neutral] tube defects. J Biomed Sci. 2011;18:66.

35. Usui T, Shima Y, Shimada Y, Hirano S, Burgess RW, Schwarz TL, Takeichi M, Uemura T. Flamingo, a seven-pass transmembrane cadherin, regulates planar cell polarity under the control of frizzled. Cell. 1999;98(5):585-95.

36. Robinson A, Escuin S, Doudney K, Vekemans M, Stevenson RE, Greene ND, Copp AJ, Stanier P. Mutations in the planar cell polarity genes CELSR1 and SCRIB are associated with the severe neural tube defect craniorachischisis. Hum Mutat. 2012;33(2):440-7.

37. Allache R, De Marco P, Merello E, Capra V, Kibar Z. Role of the planar cell polarity gene CELSR1 in neural tube defects and caudal agenesis. Birth Defects Res A Clin Mol Teratol. 2012;94(3):176-81.

38. Qu Y, Huang Y, Feng J, Alvarez-Bolado G, Grove EA, Yang Y, Tissir F, Zhou L, Goffinet AM. Genetic evidence that Celsr3 and Celsr2, together with Fzd3, regulate forebrain wiring in a Vangl-independent manner. Proc Natl Acad Sci U S A. 2014;111(29):E2996-3004.

Submit your next manuscript to BioMed Central and we will help you at every step:

- We accept pre-submission inquiries

- Our selector tool helps you to find the most relevant journal

- We provide round the clock customer support

- Convenient online submission

- Thorough peer review

- Inclusion in PubMed and all major indexing services

- Maximum visibility for your research

Submit your manuscript at www.biomedcentral.com/submit 